\title{
Effect of PTEN Polymorphism on the Development of Hepatitis B Virus-asso- ciated Hepatocellular Carcinoma
}

\author{
Soon Sun Kim', Jung Woo Eun', Hyo Jung Cho', Hyun-Young Lee ${ }^{2}$, Chul Won Seo ${ }^{3}$, Gil Ho Lee', So Young Yoon', \\ Choong Kyun Noh', Sung Won Cho', Jae Youn Cheong ${ }^{1}$ \\ ${ }^{1}$ Department of Gastroenterology, Ajou University School of Medicine; ${ }^{2}$ Clinical Trial Center, Ajou University Hospital; ${ }^{3}$ Department of Bio- \\ medical Sciences, Ajou University Graduate School of Medicine, Suwon, Korea
}

Received Jan. 30, 2019

Revised Feb. 26, 2019

Accepted Feb. 26, 2019
Background/Aims: Phosphatase and tensin homolog (PTEN) is a known tumor suppressor gene that is downregulated in hepatocellular carcinoma (HCC). Here, we investigated the association between single nucleotide polymorphisms (SNPs) of PTEN and HCC development in patients with hepatitis B virus (HBV) infection.

Methods: Six SNPs of PTEN at positions rs1234221, rs1903860, rs1234220, rs1903858, rs2299941, and rs17431184 were analyzed in a development population (417 chronic HBV carriers without HCC and 281 chronic HBV carriers with HCC). PTEN rs1903858, rs1903860, and rs2299941 SNPs were further assessed for the development of $\mathrm{HCC}$ in a validation population of 200 patients with HBV-related liver cirrhosis.

Results: In the development population, PTEN rs1903860 C allele, rs1903858 G allele, and rs2299941 G allele were associated with a low risk of HCC. The haplotype A-T-A-A-A was associated with an increased risk of HCC (recessive model; odds ratio $=2.277,95 \%$ confidence interval $[C I]=1.144-4.532, P=0.019$ ). In the validation population, $P T E N$ rs $2299941 \mathrm{G}$ allele was the only significant protective genetic polymorphism related to $\mathrm{HCC}$ development after adjustment for age and sex (hazard ratio $=0.582,95 \% \mathrm{Cl}=0.353-0.962, P=0.035$ ).

Conclusions: These findings suggest that genetic polymorphisms in PTEN may affect HCC development in patients with chronic HBV infection. (J Liver Cancer 2019;19:46-54)

Keywords: PTEN; Polymorphism, single nucleotide; Hepatocellular carcinoma; Hepatitis B virus

\section{INTRODUCTION}

Hepatocellular carcinoma (HCC) is one of the most predominant malignancies worldwide. HCC is the fifth most common cancer in men, seventh most common in women,

\footnotetext{
Corresponding author : Jae Youn Cheong

Department of Gastroenterology, Ajou University School of Medicine, 164 World cup-ro, Yeongtong-Gu, Suwon 16499, Korea

Tel. +82-31-219-6939, Fax. +82-31-219-5999

E-mail; jaeyoun620@gmail.com

https://orcid.org/0000-0001-6246-1783
}

and third most common cause of cancer-related deaths globally. ${ }^{1}$ Hepatitis B virus (HBV) infection is one of the most significant causes of HCC, and genetic alterations in HBV virus were reported to be associated with HCC development and characteristics. ${ }^{2,3}$ However, further research on host genetic determinants is warranted for the development of HCC in patients with chronic HBV infection.

Phosphatase and tensin homolog (PTEN) deleted on chromosome 10 is a well-known tumor suppressor in several cancer types. PTEN can dephosphorylate phosphoinositide 3-kinase, which in turn regulates protein kinase B Akt ex-

Copyright $\odot 2019$ by The Korean Liver Cancer Association. All rights reserved.

This is an Open Access article distributed under the terms of the Creative Commons Attribution Non-Commercial License (http://creativecommons.org/licenses/by-nc/3.0/) which permits unrestricted non-commercial use, distribution, and reproduction in any medium, provided the original work is properly cited. 
pression. This regulatory mechanism has downstream effects on transcription, proliferation, cell survival, invasiveness, and angiogenesis. ${ }^{4}$ PTEN is also linked with several mechanisms related to the p53 tumor suppressor. Through p53 and other mechanisms, PTEN loss leads to genomic instability. ${ }^{5}$ Any decrease in PTEN expression was shown to be correlated with increased tumor grade, advanced disease stage, and poor prognosis. ${ }^{6}$ PTEN expression is reduced in nearly half of HCC cases either via mutation or methylation, which may further reduce its expression. ${ }^{6}$ At the cellular level, $P T E N$ is a target in the pathogenic pathway of hepatitis $\mathrm{C}$ virus core protein and HBV X protein. ${ }^{7.8}$

Single nucleotide polymorphisms (SNPs) are one of the most commonly occurring forms of genetic variations in the human genome. Several SNPs in genes such as p53 and tumor necrosis factor-alpha as well as those related to Wnt/ $\beta$-catenin pathway have been associated with the risk of HCC development. ${ }^{9-11}$ However, the effects of PTEN polymorphism on HCC risk in patients with chronic HBV infection have been rarely reported. ${ }^{12}$ In the present study, we evaluated the relationship between PTEN polymorphism and HCC risk in two independent chronic HBV infection population.

\section{METHODS}

\section{Study subjects}

This study was conducted with two separate populations comprising chronic HBV carriers. To investigate the association between PTEN polymorphism and HCC, a total of 698 patients (development population, 417 chronic HBV carriers without HCC and 281 chronic HBV carriers with HCC) were enrolled in an observational case-control study. These patients had visited the outpatient clinics of the Gastroenterology Department of Ajou University Hospital between March 2002 and December 2008. Chronic HBV carriers were diagnosed as per the positive results for HBsAg and HBV DNA for more than 6 months. The multiphase computed tomography or magnetic resonance imaging of patients diagnosed with HCC showed key imaging features, including size $\geq 1 \mathrm{~cm}$, arterial phase hyperenhancement, and depending on exact size, a combination of washout, threshold growth, and capsule appearance. Liver biopsy was performed for diagnosis in cases wherein these criteria were absent but HCC or other malignancy was considered probable. ${ }^{13}$ In addition, to validate the association between PTEN SNPs and HCC development, we enrolled a retrospective population comprising 200 patients (validation population) with $\mathrm{HBV}$-associated liver cirrhosis. Liver cirrhosis was defined with histological information or ultrasonographic findings associated with cirrhosis (spleen size, $>12 \mathrm{~cm}$; portal vein, $>16 \mathrm{~mm}$; nodules within the hepatic parenchyma). ${ }^{14}$ These patients had started $0.5 \mathrm{mg}$ entecavir between September 2006 and November 2012 and received entecavir treatment for at least 6 months. Patients were monitored every 3 to 6 months for the development of HCC until February 2015. Patients that developed HCC within 6 months were excluded from the study. All investigations performed in the present study were conducted in accordance with the guidelines of the 1975 Declaration of Helsinki. Informed consent was obtained from all participants, and the study protocol was approved by the Institutional Review Board of Human Research of Ajou University Hospital (GN3-08-030).

\section{DNA preparation}

Blood samples were stored at $-80^{\circ} \mathrm{C}$ until analysis. Genomic DNA was purified with G-DEXTM blood genomic DNA purification kit (Intron Biotechnology Inc., Seongnam, South Korea) and quantified using PicoGreen double-stranded DNA quantification reagent as per the standard protocol (Molecular Probes, Eugene, OR, USA). PicoGreen working solution was prepared (1:200 dilution) in Tris-EDTA buffer (pH 8.0). The $\lambda$ DNA standard and samples were mixed with PicoGreen working solution and incubated at $25^{\circ} \mathrm{C}$ for $5 \mathrm{~min}$ utes. Plates were read using Victor ${ }^{\mathrm{TM}} 3$ multi-label counter (excitation $480 \mathrm{~nm}$ and emission $520 \mathrm{~nm}$; PerkinElmer Inc., Waltham, MA, USA). A standard DNA concentration curve was generated using known $\lambda$ DNA concentrations. 


\section{Genotyping}

The validated SNPs in PTEN were identified using a public SNP database (http://www.ncbi.nlm.nih.gov/projects/SNP/). For the development population, we chose six SNPs with minor allele frequency $\geq 5 \%$ and $\mathrm{r}^{2} \geq 0.8$, as per the literature review. Thus, we analyzed the SNPs at six polymorphic sites, namely, positions rs1234221 (A to C substitution), rs1903860 ( $\mathrm{T}$ to $\mathrm{C}$ substitution), rs1234220 (A to G substitution), rs1903858 (A to G substitution), rs2299941 (A to G substitution), and rs17431184 (T to C substitution) (Table 1). Genotyping was performed using GoldenGate genotyping assay kit according to standard protocols (Illumina Inc., San Diego, CA, USA) as previously described. ${ }^{15}$ Signal intensities were extracted and analyzed using BEADSTUDIO software ver. 3.0.22 (Illumina Inc.). For the validation population, we selected three SNPs (rs1903860, rs1903858, and rs2299941) based on the results from the development population.

\section{Statistical analysis}

Statistical analysis was performed using IBM SPSS ver. 22.0 (IBM Corp., Armonk, NY, USA). Maintenance of Hardy-Weinberg equilibrium for each SNP was investigated to determine any deviation in genotype frequency using $\chi^{2}$ tests. Genetic models used in association testing were the co-dominant, dominant, and recessive models.

We analyzed the association between genotype and HCC presence using a multiple logistic regression model by controlling for covariates such as age and gender. In this analysis, chronic HBV carriers without HCC were regarded as the control group, while their analysis results were compared with those of HBV-associated patients with HCC. For the validation population, the association between HCC development and genotype was analyzed using multivariate Cox regression analysis.

The presence of a linkage disequilibrium (LD) block of SNPs was confirmed using HAPLOVIEW software (version 4.0; http://www.broad.mit.edu/mpg/haploview). Individual haplotypes were inferred using the EM algorithm with the SAS haplotype procedure. Haplotype analysis was performed using multiple logistic regression. All statistical tests were two-tailed and statistical significance was set at $P<0.05$. For more precise estimates, statistical computation was used to determine $P$ values of Fisher's exact test with Monte Carlo simulation (number of iteration $=100,000$ ).

\section{RESULTS}

\section{Patients characteristics}

Baseline demographic and clinical parameters of development population were shown as chronic HBV carriers without HCC and patients with HBV-associated HCC (Table 2). Patients with HCC were older than chronic HBV carriers without HCC, and men predominated both groups. Clinicopathologic characteristics that estimated the severity of cancer among the enrolled patients with HCC are depicted in Table 2. Serum levels of alpha-fetoprotein greater than 400 $\mathrm{ng} / \mathrm{mL}$ were detected in approximately one-third of patients. In total, 108 patients (44.5\%) had HCC larger than $5 \mathrm{~cm}$ in diameter, and 77 patients (31.8\%) exhibited vascular inva-

Table 1. SNP information of PTEN analyzed in this study

\begin{tabular}{|c|c|c|c|c|c|c|}
\hline Gene & Reference sequence & HWE & Major & Minor & Location & Position \\
\hline PTEN (-7748A/C) & rs1234221 & 0.048 & A & C & 10q23.31 & Downstream \\
\hline PTEN $(-4017 C / T)$ & rs1903860 & 0.006 & $\mathrm{~T}$ & C & $10 q 23.31$ & 3'-UTR \\
\hline PTEN (INT1) & rs1234220 & 0.109 & A & G & 10q23.31 & Intron \\
\hline PTEN (IVS1-97A/G) & rs1903858 & 0.425 & A & G & $10 \mathrm{q} 23.31$ & Intron (boundary) \\
\hline PTEN (IVS5) & rs2299941 & 0.008 & A & G & g0q23.31 & Intron \\
\hline PTEN (IVS7-400C/T) & rs17431184 & $<0.001$ & $\mathrm{~T}$ & C & $10 q 23.31$ & Intron \\
\hline
\end{tabular}

SNP, single nucleotide polymorphism; PTEN, phosphatase and tensin homolog; HWE, Hardy-Weinberg equilibrium. 
sion. About half of patients (54.2\%) presented advanced tumor stage (modified Union for International Cancer Control stage III + IV).

Baseline characteristics of validation population are shown in Table 3. About one-third of the validation population was detected positive for hepatitis B e-antigen, and 20\% patients had decompensated cirrhosis. In total, 41 patients from the validation population developed HCC during the course of entecavir therapy. The mean HCC size was $2.8 \pm 2.4 \mathrm{~cm}$, while the mean number of HCC was $1.3 \pm 0.5$.

\section{Genotype distribution and haplotype con- struction}

The observed genotype frequencies of all six SNPs of PTEN in patients with HCC as well as chronic HBV carriers without HCC were in agreement with the Hardy-Weinberg equilibrium $(P>0.05)$. These SNPs were analyzed for LD, and their haplotypes were constructed. From EM algorithm, four common haplotypes, namely, ht1 (A-C-A-G-G), ht2 (A-TA-A-A), ht3 (C-T-A-A-A), ht4 (A-T-G-G-A), and another

Table 2. Baseline characteristics of the development population

\begin{tabular}{lcc}
\hline & \multicolumn{2}{c}{ Development population } \\
\cline { 2 - 3 } Characteristic & $\begin{array}{c}\text { Chronic HBV } \\
\text { carriers with } \\
\text { HCC (n=281) }\end{array}$ & $\begin{array}{c}\text { Chronic HBV } \\
\text { carriers without } \\
\text { HCC (n=417) }\end{array}$ \\
\hline Age (years) & $53.6 \pm 10.2$ & $39.7 \pm 9.6$ \\
Male & $215(76.5)$ & $334(80.1)$ \\
Alpha fetoprotein & \\
$>400$ ng/mL & $87(36.0)$ & \\
Tumor size & $108(44.6)$ & \\
$>5$ cm & $77(31.8)$ & \\
Major vessel invasion & \\
Tumor stage (modified & \\
UICC) & \\
I & $27(11.2)$ \\
II & $84(34.7)$ \\
III & $87(36.0)$ \\
IV & $44(18.2)$ \\
\hline
\end{tabular}

Values are presented as mean \pm standard deviation or number (\%). HBV, hepatitis B virus; HCC, hepatocellular carcinoma; UICC, Union for International Cancer Control. rare haplotype (ht5) at rs1234221, rs1903860, rs1234220, rs1903858, and rs2299941 positions of PTEN were estimated. The values of LD coefficient (D') ranged from 0.859 to 1.000 , and those of $\mathrm{r}^{2}$ ranged from 0.046 to 0.811 . The most common haplotype was ht1 (A-C-A-G-G) (Fig. 1A). For the validation population, four common haplotypes T-A-A, C-G-G, T-G-A, and G-G-A at rs1903860, rs1903858, and rs2299941 positions were estimated. The values of $\left(D^{\prime}\right)$ ranged from 0.986 to 1.000 , while those of $r^{2}$ ranged from 0.553 to 0.833 (Fig. 1B).

\section{Association between genotype/haplotype frequency and presence of HCC in the devel- opment population}

The relationship between development of HCC and genotype/haplotype frequencies of the six SNPs in PTEN was investigated among chronic HBV carriers (Table 4). PTEN rs 1903860 C allele was associated with decreased risk of HCC development (odds ratio $[\mathrm{OR}]=0.829,95 \%$ confidence interval $[\mathrm{CI}]=0.428-0.980, P=0.040)$ in the co-dominant model. PTEN rs $1903858 \mathrm{G}$ allele was associated with decreased risk

Table 3. Baseline characteristics of the validation population

\begin{tabular}{|c|c|}
\hline Characteristic & Total $(n=200)$ \\
\hline Age (years) & $50.0 \pm 8.5$ \\
\hline Male & $141(70.1)$ \\
\hline Decompensated cirrhosis & $41(20.4)$ \\
\hline Diabetes mellitus & $25(12.4)$ \\
\hline HBeAg positivity & $67(33.3)$ \\
\hline Albumin (g/dL) & $3.8 \pm 0.7$ \\
\hline Bilirubin (mg/dL) & $1.8 \pm 1.8$ \\
\hline ALT (IU/L) & $146.5 \pm 275.1$ \\
\hline Platelet count $\left(\times 10^{9} / \mathrm{L}\right)$ & $116.6 \pm 59.4$ \\
\hline INR & $1.2 \pm 0.3$ \\
\hline AFP (ng/mL) & $33.9 \pm 67.8$ \\
\hline HBV DNA $\left(\log _{10} \mid \mathrm{U} / \mathrm{mL}\right)$ & $6.0 \pm 1.4$ \\
\hline Follow-up duration (month) & $58.8(6-100)$ \\
\hline \multicolumn{2}{|c|}{$\begin{array}{l}\text { Values are expressed as mean } \pm \text { standard deviation; median (range } \\
\text { or number (\%). } \\
\text { HBeAg, hepatitis B e-antigen; ALT, alanine aminotransferase; INF } \\
\text { international normalized ratio; AFP, alpha-fetoprotein; HBV, hepatit } \\
\text { B virus. }\end{array}$} \\
\hline
\end{tabular}


of HCC development in the co-dominant and recessive model $(\mathrm{OR}=0.534,95 \% \mathrm{CI}=0.342-0.833, P=0.006$ and $\mathrm{OR}=0.613,95 \% \mathrm{CI}=0.406-0.927, P=0.020$, respectively). An association was also reported between PTEN rs2299941 G allele and decreased risk of HCC development in the co-dominant model $(\mathrm{OR}=0.658,95 \% \mathrm{CI}=0.436-0.992, P=0.045)$. $\mathrm{Ht} 2$ (A-T-A-A-A) was associated with increased risk of HCC $(\mathrm{OR}=2.277,95 \% \mathrm{CI}=1.144-4.532, P=0.019)$.

\section{Association between PTEN genotype/haplo- type frequency and development of HCC in the validation population}

We evaluated the relationship between PTEN rs1903860, rs1903858, and rs2299941 genotypes/haplotypes and HCC development using the validation population including patients with HBV-related liver cirrhosis. Among 200 patients, $41(20.5 \%)$ developed HCC during the follow-up period (median, 58.8 months; range, 6-100 months). One SNP and

(A)

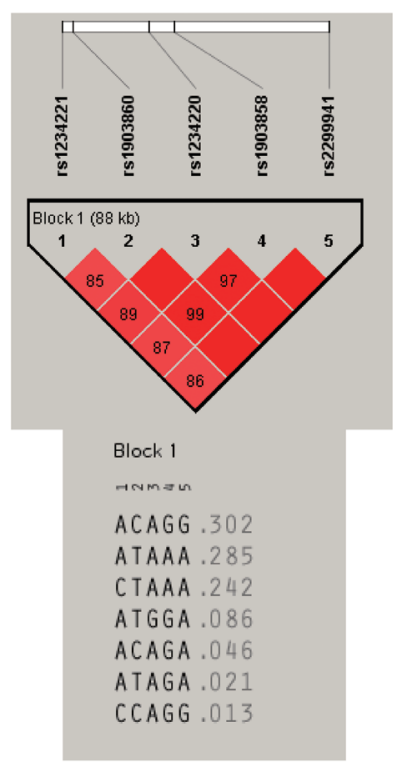

\begin{tabular}{ccccc}
\hline L1 & L2 & D' & LOD & $r^{2}$ \\
\hline rs1234221 & rs1903860 & 0.859 & 33.07 & 0.148 \\
rs1234221 & rs1234220 & 0.893 & 7.7 & 0.027 \\
rs1234221 & rs1903858 & 0.872 & 55.58 & 0.237 \\
rs1234221 & rs2299941 & 0.863 & 26.5 & 0.12 \\
rs1903860 & rs1234220 & 1 & 16.35 & 0.056 \\
rs1903860 & rs1903858 & 0.993 & 183.09 & 0.627 \\
rs1903860 & rs2299941 & 1 & 246.21 & 0.811 \\
rs1234220 & rs1903858 & 0.978 & 24.31 & 0.106 \\
rs1234220 & rs2299941 & 1 & 12.86 & 0.046 \\
rs1903858 & rs2299941 & 1 & 149.43 & 0.516 \\
\hline
\end{tabular}

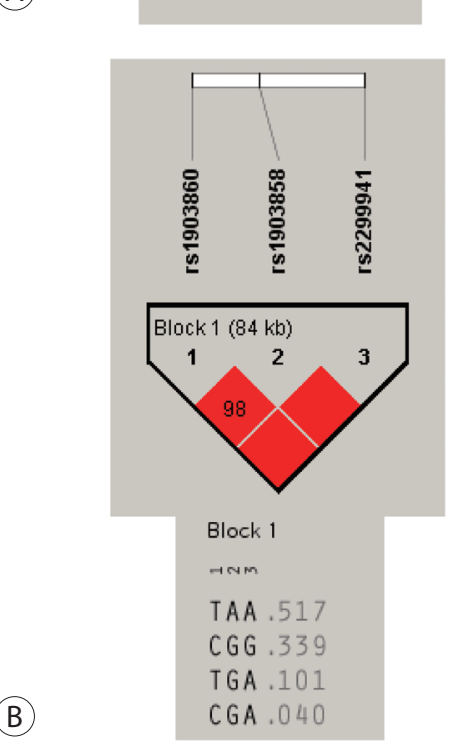

\begin{tabular}{ccccc}
\hline L1 & L2 & D' & LOD & $r^{2}$ \\
\hline rs1903860 & rs1903858 & 0.986 & 45.28 & 0.657 \\
rs1903860 & rs2299941 & 1 & 62.58 & 0.833 \\
rs1903858 & rs2299941 & 1 & 38.8 & 0.553 \\
\hline
\end{tabular}

Figure 1. Linkage disequilibrium blocks and haplotypes of PTEN. (A) Haplotypes of rs1234221, rs1903860, rs1234220, rs1903858, and rs2299941 in PTEN and linkage disequilibrium coefficient ( $D^{\prime}$ and $r^{2}$ ). (B) Haplotypes of rs1903860, rs1903858, and rs2299941 in PTEN and linkage disequilibrium coefficient $\left(D^{\prime}\right.$ and $\left.r^{2}\right)$. PTEN, phosphatase and tensin homolog. 
Table 4. Association between PTEN genotype/haplotype frequencies and HCC development in development cohort

\begin{tabular}{|c|c|c|c|c|c|c|c|c|c|}
\hline \multirow{2}{*}{$\begin{array}{l}\text { rs\# or } \\
\text { haplotype }\end{array}$} & \multirow{2}{*}{ Allele } & \multicolumn{2}{|c|}{ Co-dominant $^{*}$} & \multicolumn{2}{|c|}{ Co-dominant $^{\dagger}$} & \multicolumn{2}{|c|}{ Dominant $^{\ddagger}$} & \multicolumn{2}{|c|}{ Recessive $^{\S}$} \\
\hline & & OR $(95 \% \mathrm{Cl})$ & $P$-value & OR $(95 \% \mathrm{Cl})$ & $P$-value & OR (95\% Cl) & $P$-value & OR $(95 \% \mathrm{Cl})$ & $P$-value \\
\hline rs1234221 & $A>C$ & 0.878 (0.583-1.322) & 0.533 & $1.198(0.618-2.321)$ & 0.593 & $1.258(0.661-2.395)$ & 0.484 & 0.939 (0.642-1.373) & 0.745 \\
\hline rs1903860 & $\mathrm{T}>\mathrm{C}$ & $0.648(0.428-0.980)$ & $0.040^{\pi}$ & $0.880(0.506-1.530)$ & 0.651 & $1.087(0.649-1.820)$ & 0.751 & $0.706(0.482-1.034)$ & 0.074 \\
\hline rs1234220 & $A>G$ & $0.829(0.481-1.431)$ & 0.501 & $1.296(0.258-6.514)$ & 0.753 & $1.332(0.266-6.680)$ & 0.727 & $0.862(0.510-1.456)$ & 0.862 \\
\hline rs1903858 & $A>G$ & $0.534(0.342-0.833)$ & $0.006^{\pi}$ & $0.804(0.479-1.349)$ & 0.804 & $1.179(0.758-1.834)$ & 0.464 & $0.613(0.406-0.927)$ & $0.020^{\pi}$ \\
\hline rs2299941 & $A>G$ & $0.658(0.436-0.992)$ & $0.045^{\pi}$ & $0.925(0.503-1.699)$ & 0.801 & $1.108(0.619-1.982)$ & 0.731 & $0.714(0.488-1.044)$ & 0.082 \\
\hline rs17431184 & $\mathrm{T}>\mathrm{C}$ & $1.105(0.671-1.535)$ & 0.944 & $1.085(0.537-2.192)$ & 0.821 & $1.079(0.542-2.150)$ & 0.828 & 1.029 (0.700-1.511) & 0.885 \\
\hline $\mathrm{Ht} 1$ & ACAGG & $0.933(0.4921 .771)$ & 0.833 & $0.693(0.459-1.046)$ & 0.081 & $0.740(0.504-1.087)$ & 0.124 & $1.094(0.592-2.024)$ & 0.773 \\
\hline $\mathrm{Ht} 2$ & ATAAA & $1.798(0.862-3.750)$ & 0.118 & $0.688(0.453-1.045)$ & 0.080 & $0.778(0.519-1.168)$ & 0.227 & $2.277(1.144-4.532)$ & $0.019^{\Pi}$ \\
\hline $\mathrm{Ht} 3$ & CTAAA & $1.298(0.666-2.528)$ & 0.443 & $0.925(0.563-1.519)$ & 0.758 & $1.034(0.676-1.581)$ & 0.878 & $1.319(0.682-2.549)$ & 0.411 \\
\hline $\mathrm{Ht} 4$ & ATGGA & $1.360(0.273-6.789)$ & 0.708 & $1.132(0.620-2.065)$ & 0.687 & $1.155(0.652-2.045)$ & 0.622 & $1.341(0.269-6.679)$ & 0.721 \\
\hline $\mathrm{Ht} 5$ & Other & $5.244(0.349-78.806)$ & 0.231 & $0.944(0.518-1.721)$ & 0.851 & $1.012(0.561-1.823)$ & 0.970 & $5.275(0.351-79.201)$ & 0.229 \\
\hline
\end{tabular}

These results were adjusted with gender and age.

PTEN, phosphatase and tensin homolog; HCC, hepatocellular carcinoma; OR, odds ratio; $\mathrm{Cl}$, confidence interval.

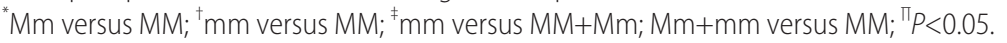

Table 5. Association between PTEN genotype/haplotype frequencies and HCC development in validation cohort

\begin{tabular}{|c|c|c|c|c|c|c|c|}
\hline \multirow{2}{*}{$\begin{array}{l}\text { rs\# or } \\
\text { haplotype }\end{array}$} & \multirow{2}{*}{ Genotype } & \multirow{2}{*}{ No HCC } & \multirow{2}{*}{$\mathrm{HCC}$} & \multicolumn{3}{|c|}{ Univariate $P$-value } & \multirow{2}{*}{$\begin{array}{c}\text { Multivariate } \\
P \text {-value }\end{array}$} \\
\hline & & & & Co-dominant & Dominant & Recessive & \\
\hline \multirow[t]{3}{*}{ rs1903860 } & TT & $54(34.2)$ & $16(41.0)$ & 0.678 & 1.000 & $0.046^{*}$ & 0.459 \\
\hline & $\mathrm{CT}$ & $82(51.9)$ & $19(48.7)$ & & & & \\
\hline & $\mathrm{CC}$ & $22(13.9)$ & $4(10.3)$ & & & & \\
\hline \multirow[t]{3}{*}{ rs1903858 } & $A A$ & $38(23.9)$ & $12(29.3)$ & 0.941 & 0.459 & 0.496 & 0.681 \\
\hline & $A G$ & $88(55.3)$ & $20(48.8)$ & & & & \\
\hline & GG & $33(20.8)$ & $9(22.0)$ & & & & \\
\hline \multirow[t]{3}{*}{ rs2299941 } & AA & $62(39.5)$ & $23(56.1)$ & 0.192 & 0.603 & 0.069 & $0.035^{*}$ \\
\hline & $A G$ & $76(48.4)$ & $14(34.1)$ & & & & \\
\hline & GG & $19(12.1)$ & $4(9.8)$ & & & & \\
\hline \multirow[t]{3}{*}{ TAA } & $+/+$ & $37(23.6)$ & $11(28.2)$ & 0.955 & 0.835 & 0.784 & 0.628 \\
\hline & $+/-$ & $87(55.4)$ & $20(51.3)$ & & & & \\
\hline & $-1-$ & $33(21.0)$ & $8(20.5)$ & & & & \\
\hline \multirow[t]{3}{*}{ CGG } & $+/+$ & $19(12.1)$ & 4 (10.3) & 0.293 & 0.118 & 0.661 & 0.675 \\
\hline & $+/-$ & $76(48.4)$ & $14(35.9)$ & & & & \\
\hline & $-1-$ & $62(39.5)$ & $21(53.8)$ & & & & \\
\hline \multirow[t]{3}{*}{ TGA } & $+/+$ & $0(0.0)$ & $1(2.6)$ & 0.108 & 0.229 & 0.060 & $0.037^{*}$ \\
\hline & $+/-$ & $35(22.3)$ & $10(25.6)$ & & & & \\
\hline & $-/-$ & $122(77.7)$ & $28(71.8)$ & & & & \\
\hline \multirow[t]{3}{*}{ CGA } & $+/+$ & $0(0.0)$ & $0(0.0)$ & 0.534 & 0.534 & NA & NA \\
\hline & $+/-$ & $3(1.9)$ & $2(5.1)$ & & & & \\
\hline & $-1-$ & $154(98.1)$ & $37(94.9)$ & & & & \\
\hline
\end{tabular}

Values are presented as number (\%) unless otherwise indicated.

PTEN, phosphatase and tensin homolog; HCC, hepatocellular carcinoma.

${ }^{*} P<0.05$. 
one haplotype were associated with low risk of HCC development (Table 5). Kaplan-Meier survival estimates revealed the association between patients with PTEN rs2299941 G allele and low risk of HCC development ( $P=0.069$, Fig. 2). Furthermore, multivariate analysis showed that patients with rs2299941 AG or GG genotype exhibited a lower risk of HCC development than those with AA genotype (hazard ratio $[\mathrm{HR}]=0.582,95 \% \mathrm{CI}=0.353-0.962, P=0.035)$. The haplotype containing rs2299941 A allele (haplotype TGA) was also associated with increased risk of HCC development in the multivariate recessive model $(\mathrm{HR}=8.969,95 \% \mathrm{CI}=1.146$ $70.188, P=0.037)$. However, the number TGA homozygote carriers was too small (only one).

\section{DISCUSSION}

In the present study, we evaluated the germline polymorphism of PTEN for the prediction of HCC development in patients with chronic HBV infection. In the development population, PTEN rs1903860, rs1903858, and rs2299941 SNPs were associated with the low risk of HCC development. Further validation of these three SNPs in the HBV-associated LC population revealed PTEN rs2299941 G allele as the only

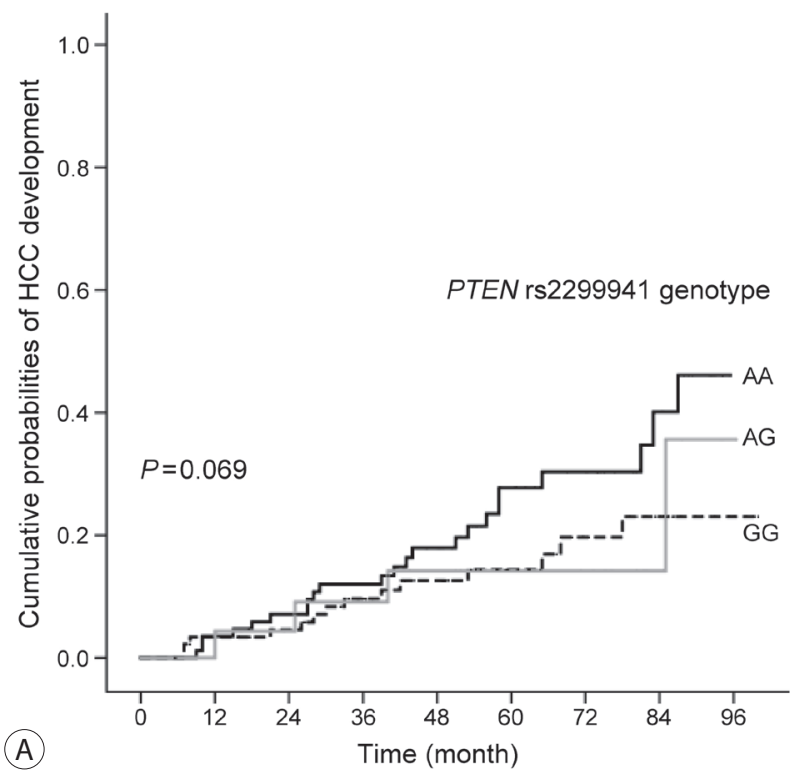

protective genetic polymorphism for HCC development in Cox regression analysis after adjustment for age and sex. Previous studies carried out in the Chinese population had not described PTEN rs2299941 SNP as a genetic locus associated with cancer, especially HCC. ${ }^{12,16}$

PTEN encodes phosphatidylinositol-3,4,5-trisphosphate 3-phosphatase, which contains a tensin-like domain and a catalytic domain similar to that of dual-specificity protein tyrosine phosphatases. Unlike most protein tyrosine phosphatases, PTEN preferentially dephosphorylates phosphoinositide substrates. Furthermore, it negatively regulates intracellular phosphatidylinositol-3,4,5-trisphosphate levels and functions as a tumor suppressor by negatively regulating the Akt signaling pathway. ${ }^{5,17-19}$ Previous studies have reported the lower expression of PTEN mRNA and protein in HCC tissues than in the adjacent non-tumor tissues. ${ }^{20-22}$ Furthermore, PTEN expression has been related to tumor aggressiveness characteristics such as pathologic grading, presence of cancer thrombus, lymph node metastasis, and prognosis in patients with HCC. ${ }^{20,21}$ However, only a few studies have been conducted on PTEN polymorphism and HCC. In the present study, we evaluated the novel SNPs of PTEN gene in HCC for the first time. PTEN rs2299941 SNP was the only significant genetic

Figure 2. Kaplan-Meier curves of cumulative probabilities of HCC development in the validation population according to (A) PTEN rs2299941 genotype and (B) haplotype TGA carrier. HCC, hepatocellular carcinoma; PTEN, phosphatase and tensin homolog.

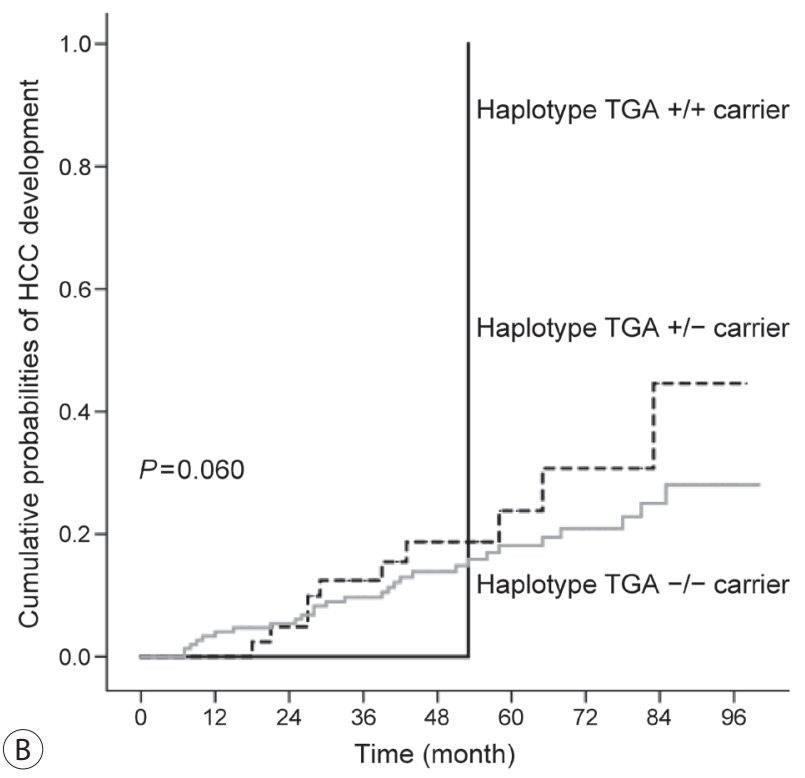


polymorphism associated with HCC development in both development and validation groups and was previously assessed in patients with prostate or endometrial cancer by other researchers. However, these studies failed to reveal any association between PTEN polymorphism and cancer. ${ }^{23,24}$ Therefore, the present study is the first report to demonstrate the relationship between PTEN rs2299941 polymorphism and cancer.

Our study has some limitations. We failed to demonstrate the direct function of PTEN rs2299941 SNP in HCC carcinogenesis. In fact, rs2299941 SNP is located within an intron and does not directly alter the protein-coding sequence of PTEN. However, the majority of reports on disease-associated mutations describing the variants that directly alter the protein-coding sequence of a gene failed to consider other mechanisms involved in gene function alterations. In cases where mutations are not found, polymorphism that resides outside of the coding region may be often overlooked. Such mechanisms include alteration in gene regulation via disruption of trans-acting factor(s) and cis-acting sequence-element interactions and may result in a pathologic state. A previous study with haplotype-based approaches reported a significant association between PTEN SNPs, including rs2299941, and Cowden and Bannayan-Riley-Ruvalcaba syndromes. ${ }^{25}$ Here, we validated the predictive ability of PTEN SNPs in a population of HBV-related liver cirrhosis. The majority of previous studies on disease-associated mutations are based on case-control study design. The present study initially evaluated six candidate PTEN SNPs in a case-control study design and then validated three PTEN SNPs in a retrospective cohort population. This is an important feature of the current study as compared to previous studies on gene polymorphism and cancer.

In conclusion, our findings demonstrate the association between genetic polymorphism in PTEN and HCC development in patients with chronic HBV infection. These results suggest the role of host genetic factors in influencing the occurrence of HBV-associated HCC.

\section{ACKNOWLEDGEMENTS}

This study was supported in part by the Scientific Research Fund of the Korean Liver Cancer Study Group (2017). This research was supported by a grant of the Korea Health Technology R\&D Project through the Korea Health Industry Development Institute (KHIDI), funded by the Ministry of Health \& Welfare, Republic of Korea (grant number: HI16C2011 \& HI18C0531). The biospecimens and data used for this study were provided by the Biobank of Ajou University Hospital, a member of Korea Biobank Network.

\section{Ethical Approval}

Informed consent was obtained from all participants, and the study protocol was approved by the Institutional Review Board of Human Research of Ajou University Hospital (GN3-08-030).

\section{Conflicts of Interest}

The authors declare no conflicts of interest.

\section{REFERENCES}

1. Bosetti C, Turati F, La Vecchia C. Hepatocellular carcinoma epidemiology. Best Pract Res Clin Gastroenterol 2014;28:753-770.

2. Lyu $H$, Lee $D$, Chung YH, Kim JA, Lee JH, Jin YJ, et al. Synergistic effects of A1896, T1653 and T1762/A1764 mutations in genotype C2 hepatitis B virus on development of hepatocellular carcinoma. J Viral Hepat 2013;20:219-224.

3. Mathews P, Lee D, Chung YH, Kim JA, Lee JH, Jin YJ, et al. Effects of genomic changes in hepatitis $B$ virus on postoperative recurrence and survival in patients with hepatocellular carcinoma. Ann Surg Oncol 2013;20:1216-1222.

4. Jiang BH, Liu LZ. PI3K/PTEN signaling in tumorigenesis and angiogenesis. Biochim Biophys Acta 2008;1784:150-158.

5. Li L, Ross AH. Why is PTEN an important tumor suppressor? J Cell Biochem 2007;102:1368-1374.

6. Wang L, Wang WL, Zhang Y, Guo SP, Zhang J, Li QL. Epigenetic and genetic alterations of PTEN in hepatocellular carcinoma. Hepatol Res 2007;37:389-396.

7. Khalid A, Hussain T, Manzoor S, Saalim M, Khaliq S. PTEN: a potential prognostic marker in virus-induced hepatocellular carcinoma. Tumour Biol 2017;39:1010428317705754. 
8. Kang-Park S, Im JH, Lee JH, Lee YI. PTEN modulates hepatitis B virus-X protein induced survival signaling in Chang liver cells. Virus Res 2006;122:53-60.

9. Jin YJ, Lee D, Chung YH, Kim JA, Kim SE, Lee YS, et al. Tumor necrosis factor-alpha gene polymorphism associated with development of hepatitis B virus-associated hepatocellular carcinoma. J Clin Gastroenterol 2015;49:e76-e81.

10. Kim SS, Cho HJ, Lee HY, Park JH, Noh CK, Shin SJ, et al. Genetic polymorphisms in the Wnt/beta-catenin pathway genes as predictors of tumor development and survival in patients with hepatitis $B$ virus-associated hepatocellular carcinoma. Clin Biochem 2016;49:792-801.

11. Liao X, Yu L, Liu X, Han C, Yu T, Qin W, et al. Genome-wide association pathway analysis to identify candidate single nucleotide polymorphisms and molecular pathways associated with TP53 expression status in HBV-related hepatocellular carcinoma. Cancer Manag Res 2018;10:953-967.

12. Du Y, Zhang YW, Pu R, Han X, Hu JP, Zhang HW, et al. Phosphatase and tensin homologue genetic polymorphisms and their interactions with viral mutations on the risk of hepatocellular carcinoma. Chin Med J (Engl) 2015;128:1005-1013.

13. Marrero JA, Kulik LM, Sirlin CB, Zhu AX, Finn RS, Abecassis MM, et al. Diagnosis, staging, and management of hepatocellular carcinoma: 2018 practice guidance by the American Association for the Study of Liver Diseases. Hepatology 2018:68:723-750.

14. Kim SS, Hwang JC, Lim SG, Ahn SJ, Cheong JY, Cho SW. Effect of virological response to entecavir on the development of hepatocellular carcinoma in hepatitis B viral cirrhotic patients: comparison between compensated and decompensated cirrhosis. Am J Gastroenterol 2014;109:1223-1233.

15. Lee SK, Kim MH, Cheong JY, Cho SW, Yang SJ, Kwack K. Integrin alpha $\mathrm{V}$ polymorphisms and haplotypes in a Korean population are associated with susceptibility to chronic hepatitis and hepatocel- lular carcinoma. Liver Int 2009;29:187-195.

16. Ding J, Gao Y, Liu R, Xu F, Liu H. Association of PTEN polymorphisms with susceptibility to hepatocellular carcinoma in a Han Chinese population. DNA Cell Biol 2011;30:229-234.

17. Cully M, You H, Levine AJ, Mak TW. Beyond PTEN mutations: the PI3K pathway as an integrator of multiple inputs during tumorigenesis. Nat Rev Cancer 2006;6:184-192.

18. Rossi DJ, Weissman IL. Pten, tumorigenesis, and stem cell selfrenewal. Cell 2006;125:229-231.

19. Sansal I, Sellers WR. The biology and clinical relevance of the PTEN tumor suppressor pathway. J Clin Oncol 2004;22:2954-2963.

20. Wan XW, Jiang M, Cao HF, He YQ, Liu SQ, Qiu XH, et al. The alteration of PTEN tumor suppressor expression and its association with the histopathological features of human primary hepatocellular carcinoma. J Cancer Res Clin Oncol 2003;129:100-106.

21. Dong-Dong L, Xi-Ran Z, Xiang-Rong C. Expression and significance of new tumor suppressor gene PTEN in primary liver cancer. J Cell Mol Med 2003;7:67-71.

22. Wu SK, Wang BJ, Yang Y, Feng XH, Zhao XP, Yang DL. Expression of PTEN, PPM1A and P-Smad2 in hepatocellular carcinomas and adjacent liver tissues. World J Gastroenterol 2007;13:4554-4559.

23. Xie CC, Lu L, Sun J, Zheng SL, Isaacs WB, Gronberg H, et al. Germline sequence variants of PTEN do not have an important role in hereditary and non-hereditary prostate cancer susceptibility. J Hum Genet 2011;56:496-502.

24. Lacey JV Jr, Yang H, Gaudet MM, Dunning A, Lissowska J, Sherman $M E$, et al. Endometrial cancer and genetic variation in PTEN, PIK3CA, AKT1, MLH1, and MSH2 within a population-based casecontrol study. Gynecol Oncol 2011;120:167-173.

25. Pezzolesi MG, Li Y, Zhou XP, Pilarski R, Shen L, Eng C. Mutationpositive and mutation-negative patients with Cowden and Bannayan-Riley-Ruvalcaba syndromes associated with distinct 10q haplotypes. Am J Hum Genet 2006;79:923-934. 